\title{
Mortalidade por suicídio no Estado do Paraná segundo meios utilizados: uma análise epidemiológica
}

Suicide mortality according to methods used in

Paraná State: an epidemiological analysis

Natalina Maria da Rosa', Rosana Rosseto de Oliveira', Guilherme Oliveira de Arruda', Thais Aidar de Freitas Mathias'

\section{RESUMO}

Objetivo: Analisar a distribuição epidemiológica da mortalidade por suicídio, segundo os meios utilizados no Estado do Paraná. Métodos: Trata-se de um estudo descritivo sobre os dados de mortalidade por suicídio e meios utilizados nas Macrorregionais de Saúde de residência, constantes no Sistema de Informações sobre Mortalidade, de 1996 a 2000 e de 2009 a 2012 e analisados por meio de estatística descritiva e inferencial, teste de qui-quadrado/ $\chi^{2}$ e medida de associação/ odds ratio. Resultados: Houve aumento na variação percentual da taxa de mortalidade por suicídio pelo enforcamento na maioria das Macrorregionais de Saúde do Estado, para ambos os sexos. Entretanto, o suicídio por arma de fogo, autointoxicação por pesticidas e outros meios apresentou declínio no período de 1996 a 2000 para 2009 a 2012. O enforcamento esteve associado ao sexo masculino e à idade superior a 45 anos, no período de 1996 a 2000, e à escolaridade acima de 12

\section{Palavras-chave}

Suicídio, epidemiologia, atenção primária à saúde, saúde mental.

\section{Keywords}

Suicide, epidemiology, primary health care, mental health. anos, não ter companheiro e óbito no domicílio, para o período de 2009 a 2012. Notou-se maior variação percentual na taxa de mortalidade por suicídio na Macrorregional Oeste. Conclusão: $\bigcirc$ levantamento das características específicas dos meios utilizados para o suicídio no Estado é um componente necessário para a implementação de intervenções preventivas para sua redução.

\section{ABSTRACT}

Objective: To analyze the epidemiological distribution of suicide mortality, according to the methods used in the state of Paraná. Methods: This is a descriptive study of suicide mortality and methods used in Health Macro-regional of residence listed in the Mortality Information System, 1996-2000 and 2009-2012, and analyzed by descriptive and inferential statistics, chisquare test $/ \chi^{2}$ and association measurement/Odds Ratio. Results: There was an increase in the percentage variation of the suicide mortality rate by hanging in the most of the State Health Macro-regionals, for both sexes. However, suicide by firearm, autointoxication by pesticides and other methods showed decline from the period 1996-2000 to 2009-2012. The hanging was associated with male gender and age over 45 years, from 1996 to 2000, and to education over 12 years, not having a partner and death at home, for the period 2009 to 2012. It was observed greater percentage variation in suicide mortality rate in the West Macro-Region. Conclusion: The survey of the specific characteristics of suicide mortality methods in the state is a necessary component for the implementation of preventive interventions to reduce suicide.

1 Universidade Estadual de Maringá (UEM), Programa de Pós-Graduação em Enfermagem.

Endereço para correspondência: Natalina Maria da Rosa

Rua Pioneiro Antonio Bughi, 468,

Jardim Paris VI

87083-720 - Maringá, PR, Brasil

E-mail: natalina_sula@hotmail.com 


\section{INTRODUÇÃO}

O suicídio é um grave problema de saúde pública mundial, com a ocorrência de aproximadamente 900 mil óbitos por ano'. Está diretamente associado à utilização de meios de maior grau de letalidade como enforcamento, arma de fogo, precipitação de lugares elevados², além do persuasivo efeito Werther (óbitos por influência de divulgação na mídia) $)^{3,4}$.

A tendência da ocorrência de óbitos por suicídio varia de acordo com o local em análise. Estudo desenvolvido na Alemanha pelo Federal Statistical Office of Germany constatou tendência decrescente nas taxas de mortalidade por suicídio entre os anos de 1998 e 2010, em virtude do grau de letalidade do meio escolhido. Meios menos letais como autointoxicação (por exemplo, pesticidas) e afogamento foram os mais utilizados ${ }^{3}$.

Estudo comparativo revela que na China a autointoxicação por pesticida representou $73,5 \%$ de todos os meios para o suicídio, comumente mais utilizados por adultos jovens de 15 a 34 anos de idade, residentes em zonas rurais ${ }^{5}$. Em contrapartida, nos Estados Unidos da América (EUA), o Web-Based Injury Statistics Query and Reporting System (WISQARS ${ }^{\mathrm{TM}}$ ) verificou crescimento da taxa de mortalidade por suicídio entre 2000 e 2010 (10,4-12,1 por 100.000 habitantes, respectivamente) decorrente do aumento de enforcamentos em 52\%. Assim como nos EUA, o Brasil também apresentou crescimento significativo de $62,5 \%$ na taxa de mortalidade por suicídio entre

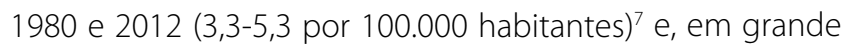
parte desse período (1980 a 2006), o enforcamento foi o principal meio utilizado pelos suicidas².

Para a Organização Mundial da Saúde (OMS), a maioria dos suicídios pode ser prevenível. O Suicide Prevention Program (SUPRE) foi uma iniciativa da OMS para reduzir o número de vidas perdidas por esse agravo. Globalmente, a partir desse manual foram elaboradas estratégias de prevenção e controle do suicídio. No Brasil, as Diretrizes Nacionais para a Prevenção do Suicídio foram implementadas segundo as particularidades governamentais e populacionais de cada Unidade Federada (UF) ${ }^{8}$.

Ressalta-se, contudo, que o planejamento estratégico de prevenção e controle do suicídio não é uma realidade para a maioria das UF, inclusive para o Paraná. Desse modo, o reconhecimento das mudanças emergentes nos meios utilizados pelos suicidas e os possíveis efeitos destes sobre as taxas de suicídio são de particular importância, na medida em que servem como base para a formulação de estratégias para sua prevenção. Apesar disso, poucos estudos com esse objetivo foram realizados no Brasil. Nesse sentido, este estudo teve por objetivo analisar a distribuição epidemiológica da mortalidade por suicídio, segundo os meios utilizados no Estado do Paraná, de 1996 a 2012.

\section{MÉTODOS}

Trata-se de um estudo epidemiológico, descritivo, no qual foram analisados os registros relacionados ao meio utilizado para o suicídio no Estado do Paraná, referentes ao período de 1996 a 2012, constantes no banco de dados do Sistema de Informações sobre Mortalidade (SIM) e dados das estimativas populacionais do Instituto Brasileiro de Geografia e Estatística (IBGE), ambos disponíveis no sítio eletrônico do Departamento de Informática do Sistema Único de Saúde (Datasus).

O Paraná, localizado na região sul do Brasil, é constituído por 399 municípios, que estão agrupados em quatro Macrorregionais de Saúde (Leste, Norte, Noroeste e Oeste). Nesse cenário, a população deste estudo constituiu-se dos suicídios de residentes nas quatro Macrorregionais de Saúde durante os 17 anos pesquisados. Foram analisados os óbitos por suicídios (ou lesões autoprovocadas) de indivíduos com idade igual ou superior a 15 anos. Para verificar a variação percentual e a distribuição da mortalidade por suicídio, de acordo com os meios utilizados, optou-se por se considerar apenas os extremos do período estudado: primeiro quinquênio de 1996 a 2000 e último quadriênio, entre 2009 e 2012.

Os dados sobre suicídio foram codificados de acordo com as categorias diagnósticas da 10a revisão da Classificação Estatística Internacional de Doenças e Problemas Relacionados à Saúde - CID-10, constantes no capítulo XX, referente às causas externas de morbidade e de mortalidade (códigos X60-X84), todas sob o título "lesões autoprovocadas intencionalmente" ${ }^{\prime \prime}$. Os meios utilizados para o suicídio foram agrupados em quatro categorias: autointoxicação por pesticida (X68); enforcamento (X70); arma de fogo (X72-X75) e outros meios (medicamentos e substâncias farmacológicas: X60-X64 e X66 e X67; gases e produto químico: X65 e X69; afogamento: $X 71$; fogo e objetos quentes: $X 76$ e $X 77$; objeto cortante e contundente: $X 78$ e X79; precipitação de lugar elevado: $\mathbf{X 0}$; objeto em movimento e impacto de veículo a motor: X81 e X82; meios especificados ou não: X83 e X84.

Foram analisadas variáveis sociodemográficas: sexo (masculino e feminino), idade (15 a 44, 45 a 64, e 65 anos e mais), escolaridade (nenhuma, 1 a 7, 8 a 11, e 12 anos e mais), situação conjugal (com companheiro, sem companheiro); e local de ocorrência dos óbitos por suicídio (ruas e áreas públicas, domicílio, e hospital). Os dados foram compilados no software Microsoft Office Excel 2013 e organizados em tabelas com frequências absolutas e relativas. Utilizou-se o teste de qui-quadrado/ $\chi^{2}$, realizado em análise bivariada no software Epi Info 7. Adotou-se como medida de associação o odds ratio (OR), com respectivo intervalo de confiança de $95 \%$, considerando-se o nível de significância de 5\%.

Calcularam-se as taxas de mortalidade por suicídio conforme meio utilizado, pela razão entre o número de óbitos por suicídio em maiores de 15 anos, e a população com ida- 
de superior a 15 anos, na mesma região e período, multiplicado por 100.000 .

Para quantificar a variação percentual dos meios utilizados para o suicídio, realizou-se a razão da subtração entre as taxas de 1996 a 2000 e 2009 a 2012, pela taxa de 1996 a 2000, multiplicado por 100. Com o número de óbitos e a população para cada período (1996 a 2000 e 2009 a 2012), geraram-se tabelas de contingência $2 \times 2$, em que o óbito para cada meio utilizado para o suicídio foi considerado a exposição, e a população que não foi a óbito (população total menos o número de óbitos por cada meio utilizado para o suicídio), a não exposição. $O$ teste qui-quadrado/ $\chi^{2}$ foi utilizado, então, para analisar a associação entre as variáveis independentes e os óbitos por suicídio, determinando-se as diferenças entre as taxas como significantes quando $p<0,05$.

Por se tratar de uma pesquisa com dados secundários, foi solicitada a dispensa do termo de consentimento livre e esclarecido ao Comitê Permanente de Ética em Pesquisa Envolvendo Seres Humanos da Universidade Estadual de Maringá (COPEP/UEM), com aprovação sob o parecer 781.253/2014.

\section{RESULTADOS}

No Estado do Paraná, durante o período estudado, 10.344 pessoas tiveram o suicídio como registro de causa básica de óbito. Em dados não documentados em tabelas, verificou-se que alguns campos do banco de dados do SIM apresentaram incompletude em suas informações. Dentre esses campos, destaca-se a ausência de 3.419 (33,1\%) dados referentes a escolaridade, 457 (4,4\%) do local de ocorrência e 320 (3,1\%) dados de situação conjugal. Ao considerar a totalidade de registros, constatou-se que a taxa média de mortalidade por suicídio no Estado do Paraná entre 1996 e 2012 foi de 8,4 óbitos por 100.000 habitantes, com redução de 19,4\%, ou seja, de 9,3 passou para 7,5 óbitos por 100.000 habitantes.

$\mathrm{Na}$ análise da variação percentual da taxa de mortalidade pelos meios utilizados para o suicídio, de 1996/2000 para 2009/2012, observou-se aumento da taxa de mortalidade por suicídio pelo enforcamento na maioria das Macrorregionais de Saúde do Estado, para ambos os sexos. Por sua vez, a variação percentual da taxa de mortalidade por suicídio pelo disparo de arma de fogo indicou redução significativa em todas as Macrorregionais, com destaque para Macrorregional Leste. Quanto à variação percentual da taxa de mortalidade por suicídio pela autointoxicação voluntária por pesticida, com exceção da Macrorregional Leste, as demais conferiram decréscimo significativo. Ao comparar as quatro Macrorregionais, a Macrorregional Oeste exibiu taxas e percentuais mais elevados para o sexo masculino e feminino, porém foi a macrorregional que obteve menor queda estatisticamente significativa na variação percentual da taxa de mortalidade por suicídio no sexo masculino (Tabela 1).
No período de 1996 a 2000, o sexo e a faixa etária apresentaram-se associados ao suicídio por enforcamento, de forma que ser do sexo masculino e ter idade superior a 45 anos apresentaram chances maiores do que o sexo feminino e a faixa etária de 15 a 44 anos. As ocorrências de suicídio que envolveram indivíduos com escolaridade igual ou superior a 12 anos de estudo, sem companheiro, o domicílio e o hospital como local de ocorrência apresentaram significativamente menores proporções e chances de enforcamento. No que se refere ao período de 2009 a 2012, constatou-se que ser do sexo masculino, não ter companheiro e o domicílio como local de ocorrência dos óbitos mostraram-se associados e com maiores chances, enquanto ter escolaridade igual a 12 anos e como local de ocorrência o hospital apresentaram chances menores para suicídio por enforcamento (Tabela 2).

Quanto ao suicídio por disparo de arma de fogo, no período de 1996 a 2000, verificaram-se maiores chances para as ocorrências que envolviam o sexo masculino, algum nível de escolaridade, ausência de companheiro e o hospital ou o domicílio como local de ocorrência da morte. Tomando-se a categoria "nenhuma escolaridade" como referência, observou-se que as demais se mostraram associadas, com incremento na razão de chance conforme aumento da escolaridade. Verificou-se semelhança no período de 2009 a 2012 em relação ao sexo e à escolaridade, porém não ocorreu o mesmo para a situação conjugal, em que não ter companheiro apresentou significativamente menor proporção e chance. O local de ocorrência, por sua vez, não se mostrou associado (Tabela 3).

Em relação ao suicídio pelo meio autointoxicação por pesticida no período de 1996 a 2000, verificou-se associação com o sexo, a faixa etária, a escolaridade e o local de ocorrência, de modo que as chances para ocorrência do suicídio foram significativamente menores para o sexo masculino, a idade igual ou superior a 65 anos e maior escolaridade, e maiores para o hospital e o domicílio como locais de ocorrência. No período de 2009 a 2012, observou-se que escolaridade entre oito e 11 anos, não ter companheiro e o domicílio como local de ocorrência do óbito representaram menores chances para o suicídio. Já o local de ocorrência permaneceu associado ao óbito por autointoxicação por pesticida, com maiores chances relacionadas ao hospital (Tabela 4).

Sobre os outros meios de suicídio, nos dois períodos estudados, as chances de ocorrência foram menores para o sexo masculino, com proporção de óbitos significativamente menor do que o sexo feminino. Também em ambos os períodos, o local de ocorrência mostrou-se associado, maior chance de ocorrência do óbito para o hospital. No período final, não ter companheiro apresentou, significativamente, maior proporção e chance de óbitos por outros meios, ao passo que o domicílio apresentou, de forma significativa, menor proporção desses óbitos, indicando assim menor chance de ocorrência (Tabela 5). 
Tabela 1. Taxas de mortalidade por suicídio, segundo sexo e meio, no Paraná e macrorregionais de saúde. Paraná, Brasil, 1996 a 2012

\begin{tabular}{|c|c|c|c|c|c|c|c|c|c|c|c|c|c|c|}
\hline \multirow{3}{*}{ Macrorregional } & \multicolumn{7}{|c|}{ Masculino } & \multicolumn{7}{|c|}{ Feminino } \\
\hline & \multicolumn{3}{|c|}{$1996 / 2000$} & \multicolumn{3}{|c|}{ 2009/2012 } & \multicolumn{4}{|c|}{$1996 / 2000$} & \multicolumn{3}{|c|}{ 2009/2012 } & \multirow[b]{2}{*}{ Var\% } \\
\hline & $\mathrm{n}$ & $\%$ & $\mathrm{Tx}$ & $\mathrm{n}$ & $\%$ & $\mathrm{Tx}$ & Var\% & $n$ & $\%$ & $\mathrm{Tx}$ & N & $\%$ & $\mathrm{Tx}$ & \\
\hline Leste & 1073 & 100 & 6,9 & 852 & 100 & 5,3 & $-23,2^{*}$ & 258 & 100 & 1,7 & 226 & 100 & 1,4 & $-15,2$ \\
\hline Enforcamento & 600 & 55,9 & 7,8 & 599 & 70,3 & 7,6 & $-2,8$ & 102 & 39,5 & 1,3 & 98 & 43,4 & 1,2 & $-7,6$ \\
\hline Arma de fogo & 289 & 26,9 & 3,8 & 98 & 11,5 & 1,2 & $-67,0^{*}$ & 69 & 26,7 & 0,9 & 27 & 11,9 & 0,3 & $-62,4^{*}$ \\
\hline Autointoxicação & 64 & 6 & 0,7 & 66 & 7,7 & 0,8 & $+0,4$ & 32 & 12,4 & 0,4 & 23 & 10,2 & 0,3 & $-30,9$ \\
\hline \multicolumn{15}{|l|}{ por pesticida } \\
\hline Outros meios & 120 & 11,2 & 1,6 & 89 & 10,4 & 1,1 & $-27,8^{*}$ & 55 & 21,3 & 0,7 & 78 & 34,5 & 0,9 & $+36,4^{*}$ \\
\hline Norte & 471 & 100 & 7,8 & 364 & 100 & 6,2 & $-19,8$ & 124 & 100 & 2 & 94 & 100 & 1,6 & $-21,4^{*}$ \\
\hline Enforcamento & 203 & 43,1 & 6,8 & 228 & 62,6 & 8 & $+17,7$ & 39 & 31,5 & 1,2 & 39 & 41,5 & 1,3 & $+2,7$ \\
\hline Arma de fogo & 102 & 21,7 & 3,4 & 51 & 14 & 1,8 & $-47,6^{*}$ & 17 & 13,7 & 0,6 & 3,0 & 3,2 & 0,1 & $-81,9^{* \#}$ \\
\hline Autointoxicação & 102 & 21,7 & 3,4 & 42 & 11,5 & 1,5 & $-56,8^{*}$ & 27 & 21,8 & 0,9 & 14 & 14,9 & 0,5 & $-46,8^{*}$ \\
\hline \multicolumn{15}{|l|}{ por pesticida } \\
\hline Outros meios & 64 & 13,6 & 2,1 & 43 & 11,8 & 1,5 & $-29,6$ & 41 & 33,1 & 1,3 & 38 & 40,4 & 1,2 & $-4,9$ \\
\hline Noroeste & 405 & 100 & 7,3 & 307 & 100 & 5,6 & $-22,8^{*}$ & 89 & 100 & 1,6 & 78 & 100 & 1,4 & $-10,7$ \\
\hline Enforcamento & 187 & 46,2 & 6,8 & 204 & 66,4 & 7,7 & $+12,3$ & 27 & 30,3 & 1,0 & 46 & 59 & 1,6 & $+71,7$ \\
\hline Arma de fogo & 83 & 20,5 & 3,0 & 39 & 12,7 & 1,5 & $-51,6^{*}$ & 6 & 6,7 & 0,2 & 2 & 2,6 & 0,1 & $-66,4$ \\
\hline Autointoxicação & 92 & 22,7 & 3,4 & 27 & 8,8 & 1,0 & $-69,8$ & 30 & 33,7 & 1,1 & 9 & 11,5 & 0,3 & $-69,8^{*}$ \\
\hline \multicolumn{15}{|l|}{ por pesticida } \\
\hline Outros meios & 43 & 10,6 & 1,6 & 37 & 12,1 & 1,4 & $-11,4$ & 26 & 29,2 & 0,9 & 21 & 26,9 & 0,7 & $-18,6$ \\
\hline Oeste & 467 & 100 & 9,4 & 407 & 100 & 8,2 & $-12,4^{*}$ & 118 & 100 & 2,4 & 96 & 100 & 1,9 & $-18,2$ \\
\hline Enforcamento & 292 & 62,5 & 11,8 & 300 & 73,7 & 12,4 & $+4,5$ & 66 & 55,9 & 2,6 & 70 & 72,9 & 2,8 & $+5,4$ \\
\hline Arma de fogo & 114 & 24,4 & 4,6 & 53 & 13 & 2,2 & $-52,7^{*}$ & 19 & 16,1 & 0,8 & 5 & 5,2 & 0,2 & $-73,9^{*}$ \\
\hline Autointoxicação & 32 & 6,9 & 1,3 & 14 & 3,4 & 0,6 & $-55,5^{*}$ & 12 & 10,2 & 0,5 & 4 & 4,2 & 0,2 & $-66,9^{* *}$ \\
\hline \multicolumn{15}{|l|}{ por pesticida } \\
\hline Outros meios & 29 & 6,2 & 1,2 & 40 & 9,8 & 1,7 & $+40,3^{*}$ & 21 & 17,8 & 0,8 & 17 & 17,7 & 0,7 & $-19,6^{*}$ \\
\hline Paraná & 2352 & 100 & 14,9 & 1930 & 100 & 12,2 & $-17,7^{*}$ & 589 & 100 & 3,6 & 494 & 100 & 3,0 & $-17,3^{*}$ \\
\hline Enforcamento & 1282 & 54,5 & 8,1 & 1331 & 69,0 & 8,4 & $+4,0$ & 234 & 39,7 & 1,4 & 253 & 51,2 & 1,5 & $+6,6$ \\
\hline Arma de fogo & 588 & 25,0 & 3,7 & 241 & 12,5 & 1,5 & $-58,9^{*}$ & 111 & 18,8 & 0,7 & 37 & 7,5 & 0,2 & $-67,1^{*}$ \\
\hline Autointoxicação & 226 & 9,6 & 1,4 & 149 & 7,7 & 0,9 & $-33,9^{*}$ & 101 & 17,1 & 0,6 & 50 & 10,1 & 0,3 & $-51,2^{*}$ \\
\hline \multicolumn{15}{|l|}{ por pesticida } \\
\hline Outros meios & 256 & 10,9 & 1,6 & 209 & 10,8 & 1,3 & $-18,1^{*}$ & 143 & 24,3 & 0,8 & 154 & 31,2 & 0,9 & $+6,2$ \\
\hline
\end{tabular}

* Significância estatística $(p<0,05)$; Teste Exato Fisher.

Tx: taxa; Var\%: variação percentual das taxas de mortalidade por suicídio.

\section{DISCUSSÃO}

O resultado da análise epidemiológica da mortalidade por suicídio entre os residentes do Estado do Paraná mostrou variações no meio utilizado para o suicídio entre os períodos de 1996 a 2000 e 2009 a 2012. O conhecimento dessas variações pode servir de subsídio aos gestores de políticas públicas do Estado, na implementação de estratégias mais eficazes para dificultar o acesso dos residentes aos meios mais utilizados para o suicídio. Estudo sobre óbitos por causas externas no Paraná reforça que a análise da distribuição da mortalidade deve nortear as ações preventivas nos diversos setores e níveis governamentais ${ }^{10}$. 
Tabela 2. Mortalidade por suicídio decorrente de enforcamento, segundo características sociodemográficas e local de ocorrência do óbito. Paraná, Brasil, 1996 a 2012

\begin{tabular}{|c|c|c|c|c|c|c|c|c|}
\hline \multirow{2}{*}{ Características } & \multicolumn{4}{|c|}{ 1996/2000 } & \multicolumn{4}{|c|}{ 2009/2012 } \\
\hline & $n$ & $\%$ & $p$ & OR (IC95\%) & $\mathrm{n}$ & $\%$ & $p$ & OR (IC95\%) \\
\hline Sexo & 1516 & & & & 1584 & & & \\
\hline Feminino & 234 & 39,7 & - & 1 & 253 & 51,2 & - & 1 \\
\hline Masculino & 1282 & 53,1 & $<0,001$ & $1,7(1,4-2,0)$ & 1331 & 69,0 & $<0,001$ & $2,1(1,7-2,5)$ \\
\hline Idade & 1854 & & & & 1584 & & & \\
\hline 15 a 44 anos & 1149 & 52,5 & - & 1 & 983 & 66,3 & - & 1 \\
\hline 45 a 64 anos & 512 & 61,0 & $<0,001$ & $1,4(1,2-1,6)$ & 434 & 62,8 & 0,113 & $0,8(0,7-1,0)$ \\
\hline 65 anos e mais & 193 & 61,5 & 0,002 & $1,4(1,1-1,8)$ & 167 & 66,8 & 0,873 & $1,0(0,7-1,3)$ \\
\hline Escolaridade & 471 & & & & 1514 & & & \\
\hline Nenhuma & 143 & 50,9 & - & 1 & 78 & 66,7 & - & 1 \\
\hline 1 a 7 anos & 261 & 57,4 & 0,086 & $1,2(0,9-1,7)$ & 942 & 68,1 & 0,747 & $1,0(0,7-1,5)$ \\
\hline 8 a 11 anos & 31 & 45,6 & 0,432 & $0,8(0,4-1,3)$ & 362 & 67,3 & 0,897 & $1,0(0,6-1,5)$ \\
\hline 12 anos e mais & 36 & 35,3 & 0,006 & $0,5(0,3-0,8)$ & 132 & 50,4 & 0,003 & $0,5(0,3-0,7)$ \\
\hline Situação conjugal & 1452 & & & & 1565 & & & \\
\hline Com companheiro & 698 & 58,9 & - & 1 & 549 & 69,7 & - & 1 \\
\hline Sem companheiro & 754 & 53,8 & 0,009 & $0,8(0,6-0,9)$ & 1016 & 75,8 & 0,002 & $1,3(1,1-1,6)$ \\
\hline Local de ocorrência & 1298 & & & & 1582 & & & \\
\hline Ruas e áreas públicas & 874 & 77,6 & - & 1 & 344 & 65,4 & - & 1 \\
\hline Domicílio & 370 & 43,4 & $<0,001$ & $0,2(0,1-0,2)$ & 1175 & 78,5 & $<0,001$ & $1,9(1,5-2,4)$ \\
\hline Hospital & 54 & 9,2 & $<0,001$ & $0,0(0,0-0,0)$ & 63 & 15,8 & $<0,001$ & $0,0(0,0-0,1)$ \\
\hline
\end{tabular}

OR: odds ratio; IC95\%: intervalo de 95\% de confiança.

Tabela 3. Mortalidade por suicídio decorrente de arma de fogo, segundo características sociodemográficas e local de ocorrência do óbito. Paraná, Brasil, 1996 a 2012

\begin{tabular}{|c|c|c|c|c|c|c|c|c|}
\hline \multirow{2}{*}{ Características } & \multicolumn{4}{|c|}{ 1996/2000 } & \multicolumn{4}{|c|}{ 2009/2012 } \\
\hline & n & $\%$ & $p$ & OR (IC95\%) & $\mathrm{n}$ & $\%$ & $p$ & OR (IC95\%) \\
\hline Sexo & 699 & & & & 278 & & & \\
\hline Feminino & 111 & 18,9 & - & 1 & 37 & 7,5 & - & 1 \\
\hline Masculino & 588 & 24,3 & 0,004 & $1,3(1,1-1,7)$ & 241 & 12,5 & 0,001 & $1,7(1,2-2,5)$ \\
\hline Idade & 699 & & & & 278 & & & \\
\hline 15 a 44 anos & 518 & 23,7 & - & 1 & 169 & 11,4 & - & 1 \\
\hline 45 a 64 anos & 128 & 15,2 & $<0,001$ & $0,5(0,4-0,7)$ & 79 & 11,4 & 0,979 & $0,9(0,7-1,3)$ \\
\hline 65 anos e mais & 53 & 16,9 & 0,007 & $0,6(0,4-0,8)$ & 30 & 12,0 & 0,781 & $1,0(0,7-1,6)$ \\
\hline Escolaridade & 173 & & & & 260 & & & \\
\hline Nenhuma & 27 & 9,6 & - & 1 & 4 & 3,4 & - & 1 \\
\hline 1 a 7 anos & 85 & 18,7 & $<0,001$ & $2,1(1,3-3,4)$ & 129 & 9,3 & 0,011 & $3,4(1,2-9,4)$ \\
\hline 8 a 11 anos & 19 & 27,94 & $<0,001$ & $3,6(1,8-7,0)$ & 79 & 14,7 & $<0,001$ & $4,8(1,7-13,5)$ \\
\hline 12 anos e mais & 42 & 41,2 & $<0,001$ & $6,5(3,7-11,5)$ & 48 & 18,3 & $<0,001$ & $6,3(2,2-18,0)$ \\
\hline Situação conjugal & 676 & & & & 273 & & & \\
\hline Com companheiro & 278 & 23,4 & - & 1 & 127 & 16,1 & - & 1 \\
\hline Sem companheiro & 398 & 28,4 & 0,004 & $1,2(1,0-1,5)$ & 146 & 10,9 & $<0,001$ & $0,6(0,4-0,8)$ \\
\hline Local de ocorrência & 595 & & & & 278 & & & \\
\hline Ruas e áreas públicas & 120 & 10,6 & - & 1 & 59 & 11,2 & - & 1 \\
\hline Domicílio & 336 & 39,4 & $<0,001$ & $5,4(4,3-6,8)$ & 160 & 10,7 & 0,737 & $0,9(0,6-1,3)$ \\
\hline Hospital & 139 & 23,7 & $<0,001$ & $2,6(1,9-3,4)$ & 59 & 14,8 & 0,103 & $1,3(0,9-2,0)$ \\
\hline
\end{tabular}


Tabela 4. Mortalidade por suicídio decorrente de autointoxicação por pesticida, segundo características sociodemográficas e local de ocorrência do óbito. Paraná, Brasil, 1996 a 2012

\begin{tabular}{|c|c|c|c|c|c|c|c|c|}
\hline \multirow{2}{*}{ Características } & \multicolumn{4}{|c|}{$1996 / 2000$} & \multicolumn{4}{|c|}{ 1909/2012 } \\
\hline & $\mathrm{N}$ & $\%$ & $p$ & OR (IC95\%) & $n$ & $\%$ & $p$ & OR (IC95\%) \\
\hline Sexo & 391 & & & & 199 & & & \\
\hline Feminino & 101 & 17,5 & - & 1 & 50 & 10,1 & - & 1 \\
\hline Masculino & 290 & 12 & $<0,001$ & $0,6(0,5-0,8)$ & 149 & 7,7 & 0,082 & $0,7(0,5-1,0)$ \\
\hline Idade & 391 & & & & 199 & & & \\
\hline 15 a 44 anos & 266 & 12,1 & - & 1 & 110 & 7,4 & - & 1 \\
\hline 45 a 64 anos & 100 & 11,9 & 0,851 & $0,9(0,7-1,2)$ & 68 & 9,8 & 0,054 & $1,3(0,9-1,8)$ \\
\hline 65 anos e mais & 25 & 7,7 & 0,030 & $0,6(0,4-0,9)$ & 21 & 8,4 & 0,586 & $1,1(0,7-1,8)$ \\
\hline Escolaridade & 136 & & & & 188 & & & \\
\hline Nenhuma & 75 & 26,7 & - & 1 & 14 & 12 & - & 1 \\
\hline 1 a 7 anos & 51 & 11,2 & $<0,001$ & $0,3(0,2-0,5)$ & 128 & 9,3 & 0,336 & $0,7(0,4-1,3)$ \\
\hline 8 a 11 anos & 4 & 5,9 & $<0,001^{\#}$ & $0,1(0,0-0,4)$ & 28 & 5,2 & 0,006 & $0,4(0,2-0,7)$ \\
\hline 12 anos e mais & 6 & 5,9 & $<0,001$ & $0,1(0,0-0,4)$ & 18 & 6,9 & 0,099 & $0,5(0,2-1,1)$ \\
\hline Situação conjugal & 380 & & & & 194 & & & \\
\hline Com companheiro & 181 & 15,3 & - & 1 & 88 & 11,2 & - & 1 \\
\hline Sem companheiro & 199 & 14,2 & 0,444 & $0,9(0,7-1,1)$ & 106 & 7,9 & 0,011 & $0,6(0,5-0,9)$ \\
\hline Local de ocorrência & 336 & & & & 199 & & & \\
\hline Ruas e áreas públicas & 44 & 3,9 & - & 1 & 38 & 7,2 & - & 1 \\
\hline Domicílio & 66 & 7,7 & $<0,001$ & $2,0(1,3-3,0)$ & 45 & 3,0 & $<0,001$ & $0,3(0,2-0,6)$ \\
\hline Hospital & 226 & 38,6 & $<0,001$ & $15,4(10,9-21,7)$ & 116 & 29,1 & $<0,001$ & $5,2(3,5-7,8)$ \\
\hline
\end{tabular}

Tabela 5. Mortalidade por suicídio decorrente de outros meios, segundo características sociodemográficas e local de ocorrência do óbito. Paraná, Brasil, 1996 a 2012

\begin{tabular}{|c|c|c|c|c|c|c|c|c|}
\hline \multirow{2}{*}{ Características } & \multicolumn{4}{|c|}{$1996 / 2000$} & \multicolumn{4}{|c|}{$2009 / 2012$} \\
\hline & $\mathbf{n}$ & $\%$ & $p$ & OR (IC95\%) & $\mathbf{n}$ & $\%$ & $p$ & OR (IC95\%) \\
\hline Sexo & 399 & & & & 363 & & & \\
\hline Feminino & 143 & 24,2 & - & 1 & 154 & 31,1 & - & 1 \\
\hline Masculino & 256 & 10,6 & $<0,001$ & $0,3(0,2-0,4)$ & 209 & 10,8 & $<0,001$ & $0,2(0,2-0,3)$ \\
\hline Idade & 399 & & & & 363 & & & \\
\hline 15 a 44 anos & 256 & 11,7 & - & 1 & 221 & 14,9 & - & 1 \\
\hline 45 a 64 anos & 100 & 11,9 & 0,872 & $1,0(0,7-1,3)$ & 110 & 15,9 & 0,538 & $1,0(0,8-1,3)$ \\
\hline 65 anos e mais & 43 & 13,7 & 0,306 & $1,1(0,8-1,6)$ & 32 & 12,8 & 0,383 & $0,8(0,5-1,2)$ \\
\hline Escolaridade & 126 & & & & 338 & & & \\
\hline Nenhuma & 36 & 12,8 & - & 1 & 21 & 17,9 & - & 1 \\
\hline 1 a 7 anos & 58 & 12,6 & 0,979 & $0,9(0,6-1,5)$ & 184 & 13,3 & 0,160 & $0,7(0,4-1,1)$ \\
\hline 8 a 11 anos & 14 & 20,6 & 0,100 & $1,7(0,8-3,4)$ & 69 & 12,8 & 0,144 & $0,6(0,3-1,1)$ \\
\hline 12 anos e mais & 18 & 17,7 & 0,229 & $1,4(0,7-2,7)$ & 64 & 24,4 & 0,162 & $1,4(0,8-2,5)$ \\
\hline Situação conjugal & 80 & & & & 97 & & & \\
\hline Com companheiro & 29 & 2,5 & - & 1 & 24 & 3,0 & - & 1 \\
\hline Sem companheiro & 51 & 3,6 & 0,080 & $1,5(0,9-2,3)$ & 73 & 5,4 & 0,010 & $1,8(1,1-2,9)$ \\
\hline Local de ocorrência & 336 & & & & 362 & & & \\
\hline Ruas e áreas públicas & 88 & 7,8 & - & 1 & 85 & 16,2 & - & 1 \\
\hline Domicílio & 81 & 9,5 & 0,185 & $1,2(0,9-1,6)$ & 117 & 7,8 & $<0,001$ & $0,4(0,3-0,5)$ \\
\hline Hospital & 167 & 28,5 & $<0,001$ & $4,7(3,5,6,2)$ & 160 & 40,2 & $<0,001$ & $3,4(2,5-4,7)$ \\
\hline
\end{tabular}

OR: odds ratio; IC95\%: intervalo de 95\% de confiança. 
O Estado do Paraná apresentou taxa de mortalidade por suicídio (8,4 óbitos por 100.000 habitantes) superior à taxa nacional (5,7 óbitos por 100.000 habitantes) ${ }^{11}$ e também dos Estados da Bahia (3,9 óbitos por 100.000 habitantes) ${ }^{12}$ e São Paulo (4,6 óbitos por 100.000 habitantes) ${ }^{13}$. Embora a taxa de mortalidade por suicídio no Estado do Paraná tenha sofrido queda, ainda se mantém alta, sinalizando a necessidade de ações preventivas e assistência oportuna aos casos de risco.

Constatou-se, também, aumento das taxas de mortalidade por suicídio pelo enforcamento entre o quinquênio de 1996 a 2000 e o quadriênio de 2009 a 2012. Por outro lado, o suicídio por arma de fogo, autointoxicação por pesticidas e outros meios apresentou declínio nos períodos estudados. Ressalta-se que o acesso do indivíduo ao meio para a morte torna-se um fator contributivo para elevar a mortalidade por suicídio. Além disso, o meio utilizado para o suicídio varia de acordo com a cultura e região de cada país ${ }^{6}$.

No Paraná, o enforcamento, o disparo de arma de fogo e a autointoxicação por pesticida representaram 85,8\% dos meios utilizados para o suicídio. $O$ perfil dos meios utilizados para o suicídio entre os americanos assemelha-se ao encontrado nesta análise, pois representa 93\% do total de todos os meios ${ }^{6}$. A prevalência de suicídio por enforcamento neste estudo se justifica por ser um meio de fácil acesso e de alto grau de letalidade, achado que coaduna com a literatura 4,13,14.

Uma iniciativa pertinente de atenuação da mortalidade por suicídio concentra-se na restrição do acesso aos meios, principalmente os resultantes de atitudes impulsivas ${ }^{15}$. Nesse contexto, os resultados da presente pesquisa sugerem que regulamentos mais rigorosos para armas de fogo, pesticidas e outros meios de suicídio possam ter contribuído para o aumento do suicídio pelo enforcamento, embora sem significância estatística. Dados semelhantes a esses foram encontrados em estudo na República da Coreia, EUA e Finlândia4.

Vale ressaltar que alguns países tiveram experiências positivas de diminuição da mortalidade por suicídio a partir de estratégias nacionais simples e de intervenções locais específicas ${ }^{16}$. A exemplo disso, dispositivos de armazenamento e restrição na importação de agrotóxicos foram iniciativas do Sri Lanka; legislação mais rigorosa para a posse de arma de fogo, na Austrália; restrição do sensacionalismo da mídia, em Hong Kong, e capacitação dos profissionais da Atenção Primária à Saúde (APS) na identificação de comportamentos suicidas foi o empreendimento do Reino Unido ${ }^{15}$.

O sexo e a faixa etária mostraram-se variáveis associadas ao suicídio no Estado do Paraná, devidamente ponderados o meio utilizado e o período analisado. A constatação de que no período de 1996 a 2000 os adultos e idosos mais velhos e os indivíduos do sexo masculino tiveram maiores chances de cometer suicídio por enforcamento, além de corroborar achados de estudo de mortalidade por suicídio em idosos brasileiros ${ }^{14}$ e estatísticas de mortalidade dos EUA ${ }^{6}$, sinaliza a importância de esforços na prevenção da atenção à saúde para esses indivíduos, principalmente ao considerar que esse grupo etário possui maior determinação para morrer ${ }^{15,17}$.

Dados da International Association for Suicide Prevention (IASP) revelaram que o risco de suicídio é elevado entre os homens de meia-idade, adultos mais velhos e ainda mais agravante entre aqueles com idade acima de 75 anos. Sendo assim, os idosos têm maior intenção suicida e tendem a usar meios mais letais do que as pessoas mais jovens. Ressalta-se, ainda, que a debilidade física decorrente da idade diminui as chances de sobrevivência após a tentativa de suicídio no fim da vida ${ }^{15}$.

Exaustivamente, a literatura aponta que os principais fatores de risco para o suicídio, em especial entre os adultos mais velhos, são decorrentes de doença física, enfermidades terminais, isolamento social, problemas familiares ou sociais (aposentadoria ou a inatividade compulsória), uso de drogas de abuso ou substâncias psicoativas. Entretanto, o histórico de tentativas de suicídio anteriores e transtornos mentais (essencialmente a depressão) denota maior vulnerabilidade para o suicídio nesse estrato etário ${ }^{14,17}$. Desse modo, a depressão é, sem dúvida, o transtorno mental mais impactante e, acima de tudo, o maior responsável pelo elevado número de mortes prematuras entre adultos e idosos ${ }^{17}$.

No período de 2009 a 2012, ser do sexo masculino, não ter companheiro e o domicílio como local de ocorrência dos óbitos mostraram-se associados aos óbitos por enforcamento. Esse achado é consistente com estudo epidemiológico em Jequié na Bahia ${ }^{12}$ e em análise do comportamento da mortalidade por suicídio no México ${ }^{18}$, o qual evidenciou que grande parte dos suicídios ocorre em indivíduos sem relações afetivas estáveis. No que concerne ao local de ocorrência da morte, os mexicanos optaram pelo próprio domicílio, possivelmente, por ser um local mais acessível para consumar o ato e por causar maior impacto emocional sobre os membros da família ${ }^{18}$.

No primeiro período, indivíduos do sexo masculino com nove a 12 anos de escolaridade foram os mais acometidos pelo suicídio por arma de fogo. Realidade também foi configurada em estudo realizado nos EUA entre os anos de 2003 a $2005^{19}$. Em estudo de autópsia psicológica realizado em Antioquia ${ }^{20}$, assim como em estudo realizado no Brasil ${ }^{11}$, Minas Gerais $^{21}$, Santa Catarina ${ }^{22}$ e no presente estudo, verificou-se forte associação do suicídio por arma de fogo com o sexo masculino.

Vale salientar que, diferentemente dos paranaenses, a maioria dos brasileiros que se valeram do uso de arma de fogo para cometer o suicídio tinha baixa escolaridade e a morte ocorreu nas ruas e áreas públicas ${ }^{11}$. Em uma análise epidemiológica do suicídio no Brasil verificou-se que, em virtude do seu alto grau de letalidade, a arma de fogo, também, 
foi o segundo meio de escolha para o suicídio ${ }^{22}$, sinalizando que a implementação de estratégias de restrição do acesso a meios fatais pode ser uma medida efetiva para prevenção do suicídio ${ }^{4,15,23}$

Uma hipótese sustentável para o decréscimo da taxa de mortalidade por suicídio pelo disparo de arma de fogo no Paraná e Macrorregionais de Saúde refere-se ao rigor quanto ao registro, posse e comercialização desse artefato mediante a instauração do Estatuto do Desarmamento. Aliada a esse estatuto, a Campanha Nacional do Desarmamento, realizada desde 2004, soma esforços para conscientizar a população sobre o perigo e risco de manter uma arma em casa e, com a entrega voluntária, retirar de circulação o maior número de armas possível. Esse conjunto de medidas tenciona o declínio do índice de criminalidade e, indiretamente, contribui para a redução do suicídio ${ }^{24}$.

Suicídio por autointoxicação com pesticida esteve relacionado ao sexo masculino entre agricultores da 11 a Regional de Saúde do Paraná25, de três províncias na China ${ }^{5}$, na área rural da Coreia do Sul ${ }^{26}$ e do Sri Lanka ${ }^{23}$. Esse achado se contrapõe à realidade encontrada na presente análise, haja vista que o percentual de suicídios por autointoxicação com pesticida foi superior na população feminina e a ocorrência do óbito no hospital esteve fortemente associada a esse meio. Presume-se que os óbitos por meios de menor grau de letalidade ocorram nos hospitais, pois, ao atentarem contra a própria vida, os potenciais suicidas recebem atendimento pré-hospitalar e, posteriormente, são assistidos em uma instituição hospitalar, apresentam complicações do quadro e acabam por vir a óbito neste local27.

Uma das hipóteses que ainda justificaria a escolha da autointoxicação por pesticida como meio para o suicídio no Paraná pauta-se no fato de ser um Estado de economia predominantemente agrícola e um dos maiores consumidores de agrotóxicos com alto potencial de periculosidade. Cumpre ressaltar que, de todo agrotóxico de classes I e II utilizado no Brasil, cerca de $80 \%$ são consumidos na agricultura paranaense ${ }^{28}$. A autointoxicação por pesticida é um dos principais motivos para os elevados índices de suicídio em áreas rurais de muitos países em desenvolvimento 29 .

Quanto aos outros meios para o suicídio, a prevalência do sexo feminino encontrada neste estudo apresentou comportamento semelhante ao estudo realizado no Brasil2, na China ${ }^{30}$, em Taiwan ${ }^{31}$ e Zurique ${ }^{32}$. Nesses estudos basicamente foram considerados precipitação de lugares elevados, gases e vapores, afogamento e submersão, permanência diante de um objeto em movimento, fumaça, fogo, chamas e medicamentos.

Estudo no Sri Lanka apontou o grande número de medicamentos estocados no domicílio como uma possível causa da mortalidade por suicídio, tanto na população rural quanto urbana ${ }^{23}$. A facilidade para aquisição de fármacos contribui de forma substancial para os altos índices de intoxicação acidental e intencional. Com vistas à promoção da saúde, o governo federal, em âmbito nacional, recomenda a venda fracionada de medicamentos nas farmácias e drogarias ${ }^{33}$, particularmente de psicofármacos para transtornos mentais ${ }^{1}$.

Nesse enfoque, os resultados deste e de vários estudos $^{1,4,11,15,32}$ apontam que, para melhor análise dos aspectos relacionados à mortalidade por suicídio, é necessário entender a relação entre suicídio e as atuais mudanças psicossociais, vividas pela população, principalmente em relação ao aumento de pessoas com transtornos mentais ${ }^{17}$. Configura-se um atributo valoroso quando se busca investigar os meios emergentes utilizados para o suicídio no mundo ou em uma localidade em específico.

Considerando-se a atual conjuntura, na qual o Estado do Paraná não contempla programas de prevenção do suicídio, promulga-se a urgente necessidade de capacitar a equipe de profissionais das Redes de Atenção à Saúde em prover a identificação precoce de indivíduos da comunidade na iminência do suicídio. Dentro dessa premissa, destaca-se que, no primeiro semestre de 2016, a Secretaria Municipal de Saúde de Maringá, pertencente a 15a Regional de Saúde do Paraná - Macrorregional Noroeste, iniciou a capacitação de profissionais da área da saúde para a prevenção e posvenção do suicídio, além da instauração do primeiro comitê de prevenção do suicídio no Estado. Também, destaca-se a capacitação de voluntários para as atividades do Centro de Valorização da Vida - CW, entidade amplamente envolvida com o apoio emocional e a prevenção do suicídio ${ }^{34}$.

Vale ressaltar também que os paranaenses portadores de sofrimento psíquico podem ser assistidos tanto pela APS quanto pelos Centros de Atenção Psicossocial - CAPS. O Estado possui cerca de 142 CAPS, oferecendo uma taxa de cobertura considerada muito boa (0,83 por 100.000 habitantes) $)^{35}$. Entretanto, a distribuição espacial do CAPS não é igualitária para todo o Estado. Os residentes da Macrorregional Oeste, por exemplo, possuem baixa cobertura assistencial do CAPS, denotando ser um preditor causal do elevado percentual e taxas dos meios utilizados para o suicídio.

Diante dessa premissa, almejam-se maior envolvimento dos profissionais da APS quanto à assistência às pessoas com transtorno mental leve ou moderado e organização dos serviços em rede de atenção à saúde regionalizada, com estabelecimento de ações intersetoriais para garantir a integralidade do cuidado ${ }^{35}$

Segundo a OMS, não existe uma maneira de impedir todos os suicídios, mas a maioria pode ser evitada. A baixa adesão de muitos países a estratégias de prevenção da mortalidade por suicídio decorre do estigma social enfrentado pelas pessoas com transtornos mentais e suicidas, em especial pela falta de consciência do suicídio como um problema de saúde pública e do tabu existente em muitas sociedades 
para discuti-lo abertamente. A sensibilização de todos e a quebra dos tabus acerca do suicídio são importantes passos para os países avançarem na prevenção da mortalidade por suicídio $^{36}$

\section{CONCLUSÕES}

A partir dos resultados apresentados, o presente estudo verificou que o aumento da mortalidade por suicídio por enforcamento está relacionado principalmente com a redução significativa dos outros meios. É importante destacar que, diante dos resultados, a maior acessibilidade e o grau de letalidade inerente ao enforcamento podem explicar o aumento do número de suicídios por esse meio. Além disso, observou-se que a Macrorregional Oeste apresentou taxas e percentuais mais expressivos.

Logo, medidas de maior rigor para restringir o acesso aos meios letais para o suicídio são indispensáveis para seu controle. Diante do exposto, essa pesquisa contribuiu no sentido de apontar as mudanças emergentes nas variações percentuais entre as taxas de mortalidade pelos meios utilizados para o suicídio no Paraná, haja vista a necessidade de estudos futuros para explicar tais alterações.

Nesse sentido, o conhecimento das características potencialmente associadas à utilização dos meios para o suicídio pode direcionar intervenções preventivas e qualificar as equipes de saúde para a assistência às pessoas com risco de suicídio e/ou que necessitam de atendimento de urgência após tentativa no âmbito dos serviços de saúde. Dessa forma, acredita-se que o presente estudo constitui-se em mais um meio que subsidia o acesso à informação sobre o suicídio e seus fatores de risco, a tomada de decisão para o enfrentamento desse problema de saúde pública e a ampliação da discussão acerca do tema.

No entanto, algumas limitações estão presentes neste estudo. Uma delas diz respeito à dificuldade de encontrar estudos comparativos que contemplem análise epidemiológica da mortalidade por suicídio em outras regiões do país, sobretudo, com enfoque nos meios específicos, impossibilitando uma discussão externa mais precisa dos resultados. Acresça-se o fato de que o uso de dados obtidos do SIM apresenta incompletude de informações, o que pode ser indicativo de subnotificação ou incorreto preenchimento das declarações de óbitos.

\section{CONTRIBUIÇÕES INDIVIDUAIS}

Natalina Maria da Rosa - Contribuiu significativamente na concepção do projeto, coleta, análise e interpretação dos dados; redação do artigo; revisão crítica relevante do conteúdo intelectual e aprovação da versão final a ser publicada.
Rosana Rosseto de Oliveira - Contribuiu significativamente na análise e interpretação dos dados; redação do artigo; revisão crítica relevante do conteúdo intelectual e aprovação da versão final a ser publicada.

Guilherme Oliveira de Arruda - Contribuiu significativamente na análise e interpretação dos dados; revisão crítica relevante do conteúdo intelectual e aprovação da versão final a ser publicada.

Thais Aidar de Freitas Mathias - Contribuiu significativamente na concepção do projeto, revisão crítica relevante do conteúdo intelectual e aprovação final da versão a ser publicada.

\section{CONFLITOS DE INTERESSE}

Os autores não possuem conflitos de interesse a serem declarados.

\section{REFERÊNCIAS}

1. Brasil. Ministério da Saúde. Programa Nacional para a Saúde Mental: Plano Nacional de Prevenção do Suicídio 2013/2017. 2013. Disponível em: http://www.portaldasaude.pt/ NR/rdonlyres/9DF9A1D5-CAFC-47DD-ACBA-59F39EF4D593/0/PlanoNacionalPrevencaoSuicidio_2013_2017.pdf. Acesso em: 24 ago. 2014.

2. Minayo MC, Pinto LW, Assis SG, Cavalcante FG, Mangas RM. Trends in suicide mortality among Brazilian adults and elderly, 1980 - 2006. Rev Saude Publica. 2012;46(2):300-9.

3. Hegerl U, Mergl R, Doganay G, Reschke K, Rummel-Kluge C. Why has the continuous decline in German suicide rates stopped in 2007? PLoS One. 2013;8(8):e71589.

4. Park S, Ahn MH, Lee A, Hong JP. Associations between changes in the pattern of suicide methods and rates in Korea, the US, and Finland. Int J Ment Health Syst. 2014;8:22.

5. Jia CX, Zhang J. Characteristics of young suicides by violent methods in rural China. J Forensic Sci. 2011;56(3):674-8.

6. Baker SP, Hu G, Wilcox HC, Baker TD. Increase in suicide by hanging/suffocation in the U.S., 2000-2010. Am J Prev Med. 2013;44(2):146-9.

7. Waiselfisz JJ. Mapa da violência 2014: os jovens do Brasil. Braślila, DF: Ministério da Justiça. Flacso, 2014.

8. 'Oliveira CF, Botega NJ. Organizador. Prevenção do suicídio: manual dirigido a profissionais das equipes de saúde e mental. Braślia, DF: Ministério da Saúde, 2006.

9. World Health Organization. International statistical classification of diseases and related health problems: instruction manual. 10th rev. Geneva. 2010. v. 2. Disponível: http:// www.who.int/classifications/icd//CD10Volume2_en_2010.pdf. Acesso em: 10 abr. 2015.

10. Lozada EMK, Mathias TAF, Andrade SM, Aidar T. Informações sobre mortalidade por causas externas e eventos de intenção indeterminada, Paraná, Brasil, 1979 a 2005. Cad Saude Publica. 2009;25(1):223-8.

11. Lovisi GM, Santos SA, Legay L, Abelha L, Valencia E. Epidemiological analysis of suicide in Brazil from 1980 to 2006. Rev Bras Psiquiatr. 2009;31(supl. 2):S86-S93.

12. Souza VS, Silva LA, Lino DCSF, Alves MS, Casotti CA, Nery AA. Tentativas de suicídio e mortalidade por suicídio em um município no interior da Bahia. J Bras Psiquiatr. 2011;60(4):294-300.

13. Bando D, Brunoni AR, Fernandes TG, Benseñor IM, Lotufo PA. Suicide rates and trends in São Paulo, Brazil, according to gender, age and demographic aspects: a join point regression analysis. Rev Bras Psiquiatr. 2012;34(3):286-93.

14. Minayo MCS, Meneghel SN, Cavalcante FG. Suicide of elderly men in Brazil. Ciênc Saúde Colet. 2013;17(10):2665-74. 
15. International Association for Suicide Prevention. World suicide prevention day: suicide prevention across the globe: strengthening protective factors and instilling hope. 2012. Disponível em: https://www.iasp.info/wspd/2012_wspd.php. Acesso em: 4 out. 2014.

16. International Association for Suicide Prevention. Preventing suicide in multicultural societies. Geneva. 2011. Disponível em: http://www.iasp.info/wspd/pdf/2011_wspd_suggested_activities.pdf. Acesso em: 4 out. 2014.

17. National Alliance on Mental IIIness. New hampshire's 2011 suicide prevention annual report: suicide prevention across the Lifespan. 2011. Disponível em: www.TheConnectProgram.org/annual-reports. Acesso em: 4 out. 2014.

18. Jimenez-Ornelas R, Cardiel-Tellez L. El suicidio y su tendencia social en México: 1990-2011. Papeles de Población. 2013;19(77):205-29.

19. Rockett IR, Wang S, Stack S, De Leo D, Frost JL, Ducatman AM, et al. Race/ethnicity and potential suicide misclassification: window on a minority suicide paradox? BMC Psychiatry. 2010;10:35.

20. González A, Betancur AR, Jaramillo CL, Palacio C, Valencia JG, Aristizábal A. Suicidio y género en Antioquia (Colombia): Estudio de autopsia psicológica. Rev Colomb Psiquiatr. 2010;39(2):251-67.

21. Vidal CEA, Gontijo ECDM, Lima LA. Tentativas de suicídio: fatores prognósticos e estimativa do excesso de mortalidade. Cad Saúde Pública. 2013;29(5):175-87.

22. Portella CH, Moretti GP, Panatto AP, Rosa MI, Quevedo J, Simões PWTA. Epidemiological profile of suicide in the Santa Catarina Coal Mining Region from 1980 to 2007. Trends Psychiatry Psychother. 2013;35(2):128-33

23. Rajapakse T, Griffiths KM, Christensen H, Cotton S. A comparison of non-fatal self-poisoning among males and females, in Sri Lanka. BMC Psychiatry. 2014;14:221.

24. Brasil. Estatuto do desarmamento: Lei no 10.826, de 2003, e legislação correlata. 4. ed. Brasília, DF: (âmara dos Deputados, Edições (âmara, 2012. (Série legislação, n. 71).

25. Scardoelli MGC, Buriola AA, Oliveira MLF, Waidman MAP. Intoxicações por agrotóxicos notificadas na 11a Regional de Saúde do Estado do Paraná. Ciênc Cuid Saúde. 2011;10(3):549-55.

26. Lee WJ, Cha ES, Park ES, Kong KA, Yi JH, Son M. Deaths from pesticide poisoning in South Korea: trends over 10 years. Int Arch Occup Environ Health. 2009 Feb;82(3):365-71.
27. Botega NJ, Marín-León L, Oliveira HB, Barros MBA, Silva VF, Dalgalarrondo P. Prevalências de ideação, plano e tentativa de suicídio: um inquérito de base populacional em Campinas, São Paulo, Brasil. Cad Saúde Pública. 2009;25(12):2632-38.

28. Londres F. Agrotóxicos no Brasil: um guia para ação em defesa da vida. Rio de Janeiro: ASPTA - Assessoria e Serviços a Projetos em Agricultura Alternativa, 2011.

29. Hawton K, Ratnayeke L, Simkin S, Harriss L, Scott V. Evaluation of acceptability and use of lockable storage devices for pesticides in Sri Lanka that might assist in prevention of selfpoisoning. BMC Public Health. 2009;9:69.

30. Hu Y, Wu L, Yu X, Zhang D, Liu X, Wang Y. Analysis of injury death trends among women in Macheng City, China, 1984-2008. BMC Public Health. 2011;11:698.

31. Lin JJ, Lu TH. Trends in solids/liquids poisoning suicide rates in Taiwan: a test of the substitution hypothesis. BMC Public Health. 2011;11(712):1-7.

32. Klein SD, Bischoff, C. Schweitzer W. Suicides in the Canton of Zurich (Switzerland). Swiss Med Wkly. 2010;140:w13102.

33. Agência Nacional de Vigilância Sanitária. Medicamento fracionado: sua saúde na medida certa. 2014. Disponível em: http://www.anvisa.gov.br/hotsite/fraciona/index.htm. Acesso em: 10 set. 2014.

34. Maringá. Portal Saúde Maringá. Saúde promove capacitação de 80 funcionários com 0 tema suicídio. 2016. Disponível em: www.2.maringa.pr.gov.br. Acesso em: 23 jan. 2016.

35. Paraná. Secretaria de Estado da Saúde. A Rede de Atenção à Saúde Mental no Paraná e a competência da APS. 2014. Disponível em: http://www.saude.pr.gov.br/arquivos/File/ APSUS_-_Ed._Permanente/Oficia_8_-_Saude_Mental/Apresentacao_Rede_de_ Atenca0_a_Saude_Mental_no_Parana_e_a_competencia_da_APS.pdf. Acesso em: 4 fev. 2015.

36. World Health Organization. First WHO report on suicide prevention. 2014a. Disponível em: http://www.who.int/mediacentre/news/releases/2014/suicide-prevention-report/en/. Acesso em: 4 out. 2014 